УДК 512.628 .2

DOI https://doi.org/10.24144/2616-7700.2021.39(2).100-115

\title{
С. А. Щоголев ${ }^{1}$, В. В. Карапетров ${ }^{2}$
}

${ }^{1}$ Одеський національний університет імені I. I. Мечникова, завідувач кафедри вищої математики, професор, доктор фізико-математичних наук sergas 1959@gmail.com

ORCID: https://orcid.org/0000-0001-8025-143X

${ }^{2}$ Одеський національний університет імені I. I. Мечникова, аспірант кафедри вищої математики valentyn.karapetrov@stud. onu.edu.ua ORCID: https://orcid.org/0000-0003-1315-4968

\section{КРИТИЧНИЙ ВИПАДОК В ТЕОРІЇ МАТРИЧНИХ ДИФЕРЕНЩАЛЬНИХ РІВНЯНЬ}

\begin{abstract}
При математичному описанні різноманітних явищ і процесів, що виникають в математичній фізиці, електротехніці, економіці, доводиться мати справу з матричними диференціальними рівняннями. Тому такі рівняння $\epsilon$ актуальними как для математиків, так і для фахівців в інших галузях природознавства. В даній статті розглядається квазілінійне матричне диференціальне рівняння з коефіцієнтами, зображуваними у вигляді абсолютно та рівномірно збіжних рядів Фур'є з повільно змінними в певному сенсі коефіцієнтами та частотою (клас $F$ ). Різниці діагональних елементів матриць лінійної частини є суто уявними, тобто ми маємо справу з критичним випадком. Але між цими діагональними елементами припускаються певні співвідношення, що вказують на відсутність резонансу між власними частотами системи і частотою зовнішньої збуджуючої сили. Розглядається задача встановлення ознак існування у такого рівняння розв'язків класу $F$. За допомогою низки перетворень рівняння зводиться до рівняння некритичного випадку, і розв'язок класу $F$ цього рівняння шукається методом послідовних наближень за допомогою принципа стискуючих відображень. Потім на підставі властивостей розв'язків перетвореного рівняння робляться висновки щодо властивостей початкового рівняння.
\end{abstract}

Ключові слова: матричні диференціальні рівняння, ряди Фур'є, повільно змінні параметри.

1. Вступ. Одним з актуальних розділів теорії звичайних диференціальних рівнянь є теорія матричних диференціальних рівнянь. Такі рівняння виникають при дослідженні різноманітних процесів в математичній фізиці, електротехніці та інших галузей, та їм присвячено багато праць, в яких досліджувалась розв'язність матричних рівнянь у різних функціональних просторах, крайові задачі для матричних диференціальних рівнянь та інші проблеми [1-5]. В даній статті продовжуються дослідження, розпочаті в працях [6,7].

2. Основні позначення та означення. Нехай $G\left(\varepsilon_{0}\right)=\{t, \varepsilon: t \in \mathbb{R}, \varepsilon \in$ $\left.\left[0, \varepsilon_{0}\right], \varepsilon_{0} \in \mathbb{R}^{+}\right\}$.

Означення 1. Скажемо, що функиія $p(t, \varepsilon)$ належить до класу $S\left(m ; \varepsilon_{0}\right)$ $(m \in \mathbb{N} \cup\{0\})$, якщо виконано наступні умови

1) $p: G\left(\varepsilon_{0}\right) \rightarrow \mathbb{C}$,

2) $p(t, \varepsilon) \in C^{m}\left(G\left(\varepsilon_{0}\right)\right)$ за $t$;

Розділ 1: Математика і статистика 
3) $d^{k} p(t, \varepsilon) / d t^{k}=\varepsilon^{k} p_{k}(t, \varepsilon)(0 \leq k \leq m)$, причому

$$
\|p\|_{S\left(m, \varepsilon_{0}\right)} \stackrel{\text { def }}{=} \sum_{k=0}^{m} \sup _{G\left(\varepsilon_{0}\right)}\left|p_{k}(t, \varepsilon)\right|<+\infty \text {. }
$$

Означення 2. Скажемо, що функиія $f(t, \varepsilon, \theta(t, \varepsilon))$ належить до класу $F\left(m ; \varepsilon_{0} ; \theta\right)(m \in \mathbb{N} \cup\{0\})$, якщо иял функція зображувана у вигляді:

$$
f(t, \varepsilon, \theta(t, \varepsilon))=\sum_{n=-\infty}^{\infty} f_{n}(t, \varepsilon) \exp (i n \theta(t, \varepsilon))
$$

причому

1) $f_{n}(t, \varepsilon) \in S\left(m, \varepsilon_{0}\right)(n \in \mathbb{Z})$;

2) $\|f\|_{F\left(m ; \varepsilon_{0}, \theta\right)} \stackrel{\text { def }}{=} \sum_{n=-\infty}^{\infty}\left\|f_{n}\right\|_{S\left(m ; \varepsilon_{0}\right)}<+\infty$;

3) $\theta(t, \varepsilon)=\int_{0}^{t} \varphi(\tau, \varepsilon) d \tau, \varphi \in \mathbb{R}^{+}, \varphi \in S\left(m, \varepsilon_{0}\right), \inf _{G\left(\varepsilon_{0}\right)} \varphi(t, \varepsilon)=\varphi_{0}>0$.

Для функції $f(t, \varepsilon, \theta(t, \varepsilon))$ позначатимемо:

$$
\Gamma_{n}[f(t, \varepsilon, \theta)]=f_{n}(t, \varepsilon)=\frac{1}{2 \pi} \int_{0}^{2 \pi} f(t, \varepsilon, \theta) \exp (-i n \theta) d \theta .
$$

Множина функцій класу $F\left(m ; \varepsilon_{0} ; \theta\right)$ утворює лінійний простір, який перетворюється на повний нормований простір введенням норми $\|\cdot\|_{F\left(m ; \varepsilon_{0} ; \theta\right)}$. Має місце ланцюжок включень: $F\left(0 ; \varepsilon_{0} ; \theta\right) \supset F\left(1 ; \varepsilon_{0} ; \theta\right) \supset \ldots \supset F\left(m ; \varepsilon_{0} ; \theta\right)$.

Нехай задано дві функції класу $F\left(m ; \varepsilon_{0} ; \theta\right)$ :

$$
u(t, \varepsilon, \theta)=\sum_{n=-\infty}^{\infty} u_{n}(t, \varepsilon) \exp (i n \theta(t, \varepsilon)), v(t, \varepsilon, \theta)=\sum_{n=-\infty}^{\infty} v_{n}(t, \varepsilon) \exp (i n \theta(t, \varepsilon)) .
$$

Добуток цих функцій визначимо формулою [8]:

$$
(u v)(t, \varepsilon, \theta)=\sum_{n=-\infty}^{\infty}\left(\sum_{s=-\infty}^{\infty} u_{n-s}(t, \varepsilon) v_{s}(t, \varepsilon)\right) \exp (i n \theta(t, \varepsilon)) .
$$

Очевидно, що $u v \in F\left(m ; \varepsilon_{0} ; \theta\right)$.

Сформулюємо деякі властивості норми $\|\cdot\|_{F\left(m ; \varepsilon_{0} ; \theta\right)}$. Нехай $u, v \in F\left(m ; \varepsilon_{0} ; \theta\right)$, $k=$ const. Тоді:

1) $\|k u\|_{F\left(m ; \varepsilon_{0} ; \theta\right)}=|k| \cdot\|u\|_{F\left(m ; \varepsilon_{0} ; \theta\right)}$;

2) $\|u+v\|_{F\left(m ; \varepsilon_{0} ; \theta\right)} \leq\|u\|_{F\left(m ; \varepsilon_{0} ; \theta\right)}+\|v\|_{F\left(m ; \varepsilon_{0} ; \theta\right)}$;

3) $\|u\|_{F\left(m ; \varepsilon_{0} ; \theta\right)}=\sum_{k=0}^{m}\left\|\frac{1}{\varepsilon^{k}} \frac{\partial^{k} u}{\partial t^{k}}\right\|_{F\left(0 ; \varepsilon_{0} ; \theta\right)}$;

4) $\|u v\|_{F\left(m ; \varepsilon_{0} ; \theta\right)} \leq 2^{m}\|u\|_{F\left(m ; \varepsilon_{0} ; \theta\right)} \cdot\|v\|_{F\left(m ; \varepsilon_{0} ; \theta\right)}$.

Дійсно, при $m=0$ згідно з формулою (1) маємо: $\|u v\|_{F\left(0 ; \varepsilon_{0} ; \theta\right)} \leq\|u\|_{F\left(0 ; \varepsilon_{0} ; \theta\right)}$. · $\|v\|_{F\left(0 ; \varepsilon_{0} ; \theta\right)}$. Далі, на підставі властивостей 1) - 3):

$\|u v\|_{F\left(m ; \varepsilon_{0} ; \theta\right)}=\sum_{k=0}^{m}\left\|\frac{1}{\varepsilon^{k}} \frac{\partial^{k}(u v)}{\partial t^{k}}\right\|_{F\left(0 ; \varepsilon_{0} ; \theta\right)} \leq \sum_{k=0}^{m} \frac{1}{\varepsilon^{k}} \sum_{j=0}^{k} C_{k}^{j}\left\|\frac{\partial^{j} u}{\partial t^{j}}\right\|_{F\left(0 ; \varepsilon_{0} ; \theta\right)} \cdot\left\|\frac{\partial^{k-j} u}{\partial t^{k-j}}\right\|_{F\left(0 ; \varepsilon_{0} ; \theta\right)} \leq$

Наук. вісник Ужгород. ун-ту. 2021. Том 39, № 2 ISSN 2616-7700 (print), 2708-9568 (online) 
$\leq 2^{m}\left(\sum_{j=0}^{m} \frac{1}{\varepsilon^{j}}\left\|\frac{\partial^{j} u}{\partial t^{j}}\right\|_{F\left(0 ; \varepsilon_{0} ; \theta\right)}\right) \cdot\left(\sum_{j=0}^{m} \frac{1}{\varepsilon^{j}}\left\|\frac{\partial^{j} v}{\partial t^{j}}\right\|_{F\left(0 ; \varepsilon_{0} ; \theta\right)}\right)=2^{m}\|u\|_{F\left(m ; \varepsilon_{0} ; \theta\right)} \cdot\|v\|_{F\left(m ; \varepsilon_{0} ; \theta\right)}$.

На підставі властивості 4) можна стверджувати, що простір $F\left(m ; \varepsilon_{0} ; \theta\right)$ утворює банахову алгебру [9].

Означення 3. Скажемо, що матриия $A(t, \varepsilon)=\left(a_{j k}(t, \varepsilon)\right)_{j, k=\overline{1, N}}$ належить до класу $S_{2}\left(m ; \varepsilon_{0}\right),(m \in \mathbb{N} \cup\{0\})$, якщо $a_{j k} \in S\left(m ; \varepsilon_{0}\right)(j, k=\overline{1, N})$.

Визначимо норму:

$$
\|A(t, \varepsilon)\|_{S_{2}\left(m ; \varepsilon_{0}\right)}=\max _{1 \leq j \leq N} \sum_{k=1}^{N}\left\|a_{j k}(t, \varepsilon)\right\|_{S\left(m ; \varepsilon_{0}\right)} .
$$

Означення 4. Скажемо, що матриц,я $B(t, \varepsilon, \theta)=\left(b_{j k}(t, \varepsilon, \theta)\right)_{j, k=\overline{1, N}}$ належить до класу $F_{2}\left(m ; \varepsilon_{0} ; \theta\right)(m \in \mathbb{N} \cup\{0\})$, якщо $b_{j k}(t, \varepsilon, \theta) \in F\left(m ; \varepsilon_{0} ; \theta\right)$ $(j, k=\overline{1, N})$.

Визначимо норму

$$
\|B(t, \varepsilon, \theta)\|_{F_{2}\left(m ; \varepsilon_{0} ; \theta\right)}=\max _{1 \leq j \leq N} \sum_{k=1}^{N}\left\|b_{j k}(t, \varepsilon, \theta)\right\|_{F\left(m ; \varepsilon_{0} ; \theta\right)}
$$

Очевидно, що якщо $B_{1} \in F_{2}\left(m ; \varepsilon_{0} ; \theta\right), B_{2} \in F_{2}\left(m ; \varepsilon_{0} ; \theta\right)$, то $B_{1}+B_{2}, B_{1} B_{2} \in$ $\in F_{2}\left(m ; \varepsilon_{0} ; \theta\right)$, і виконано: $\left\|B_{1}+B_{2}\right\|_{F_{2}\left(m ; \varepsilon_{0} ; \theta\right)} \leq\left\|B_{1}\right\|_{F_{2}\left(m ; \varepsilon_{0} ; \theta\right)}+\left\|B_{2}\right\|_{F_{2}\left(m ; \varepsilon_{0} ; \theta\right)}$, $\left\|B_{1} B_{2}\right\|_{F_{2}\left(m ; \varepsilon_{0} ; \theta\right)} \leq 2^{m}\left\|B_{1}\right\|_{F_{2}\left(m ; \varepsilon_{0} ; \theta\right)} \cdot\left\|B_{2}\right\|_{F_{2}\left(m ; \varepsilon_{0} ; \theta\right)}$.

Для матриці $A=\left(a_{j k}\right)_{j, k=\overline{1, N}}$ будемо позначати:

$$
(A)_{j k}=a_{j k}, j, k=\overline{1, N}
$$

3. Постановка задачі. Розглядається матричне диференціальне рівняння:

$$
\frac{d X}{d t}=A(t, \varepsilon) X-X B(t, \varepsilon)+P(t, \varepsilon, \theta)+\mu \Phi(t, \varepsilon, \theta, X)
$$

де $X$-невідома квадратна матриця порядку $N$, що належить деякій замкненій обмеженій області $D \subset \mathbb{C}^{N \times N}$, де $\mathbb{C}^{N \times N}$-простір комплекснозначних $(N \times N)$-матриць. $A(t, \varepsilon), B(t, \varepsilon) \in S_{2}\left(m ; \varepsilon_{0}\right), P(t, \varepsilon, \theta) \in F_{2}\left(m ; \varepsilon_{0} ; \theta\right)$. Щодо матриці-функції $\Phi(t, \varepsilon, \theta, X)$ припускається, що вона належить до класу $F_{2}\left(m ; \varepsilon_{0} ; \theta\right)$ відносно $t, \varepsilon, \theta$ і неперервна за $X$ в $D . \mu \in(0,1)$ - дійсний параметр.

Позначимо $\lambda_{j}^{1}(t, \varepsilon), \lambda_{j}^{2}(t, \varepsilon)(j=\overline{1, N})$ - власні значення відповідно матриць $A(t, \varepsilon), B(t, \varepsilon)$. I припускатимемо виконання наступних умов:

$1^{0} . \inf _{G\left(\varepsilon_{0}\right)}\left|\lambda_{j}^{1}(t, \varepsilon)-\lambda_{k}^{1}(t, \varepsilon)-i n \varphi(t, \varepsilon)\right| \geq b_{0}>0$

$\inf _{G\left(\varepsilon_{0}\right)}\left|\lambda_{j}^{2}(t, \varepsilon)-\lambda_{k}^{2}(t, \varepsilon)-i n \varphi(t, \varepsilon)\right| \geq b_{0}>0 \quad \forall n \in \mathbb{Z}, j, k=\overline{1, N}, j \neq k$.

$2^{0} . \lambda_{j}^{1}(t, \varepsilon)-\lambda_{k}^{2}(t, \varepsilon)=i \omega_{j k}(t, \varepsilon), \omega_{j k}(t, \varepsilon) \in \mathbb{R}$,

$\inf _{G\left(\varepsilon_{0}\right)}\left|\omega_{j k}(t, \varepsilon)-n \varphi(t, \varepsilon)\right| \geq b_{0}>0 \forall n \in \mathbb{Z}, j, k=\overline{1, N}$.

Розділ 1: Математика і статистика 
Вивчається питання про наявність частинних розв'язків класів $F\left(m_{1} ; \varepsilon_{1} ; \theta\right)$ $m_{1} \leq m ; \varepsilon_{1} \leq \varepsilon_{0}$ рівняння (2). Умова $2^{0}$ показує, що у даному випадку ми маємо справу з критичнмим випадком, на відміну від роботи [6], де припускалося

$$
\inf _{G\left(\varepsilon_{0}\right)}\left|\operatorname{Re}\left(\lambda_{j}^{1}(t, \varepsilon)-\lambda_{k}^{2}(t, \varepsilon)\right)\right| \geq b_{0}>0 \quad(j, k=\overline{1, N}) .
$$

\section{4. Допоміжні результати.}

Лема 1. Нехай задано скалярне лінійне диференціальне рівняння 1-го порядку

$$
\frac{d x}{d t}=\lambda(t, \varepsilon) x+u(t, \varepsilon, \theta(t, \varepsilon))
$$

де $\lambda(t, \varepsilon) \in S\left(m ; \varepsilon_{0}\right), \inf _{G\left(\varepsilon_{0}\right)}|\operatorname{Re} \lambda(t, \varepsilon)|=\gamma>0, u(t, \varepsilon, \theta) \in F\left(m ; \varepsilon_{0} ; \theta\right)$. Тодi рівняння (З) має единий частинний розв'язок $x(t, \varepsilon, \theta) \in F\left(m ; \varepsilon_{0} ; \theta\right)$. Цей розв'язок даетъся формулою:

$$
x(t, \varepsilon, \theta(t, \varepsilon))=\int_{T}^{t} u(\tau, \varepsilon, \theta(\tau, \varepsilon)) \exp \left(\int_{\tau}^{t} \lambda(s, \varepsilon) d s\right) d \tau,
$$

$\partial e$

$$
T=\left\{\begin{array}{c}
-\infty, \operatorname{Re} \lambda(t, \varepsilon) \leq-\gamma<0 \\
+\infty, \operatorname{Re} \lambda(t, \varepsilon) \geq \gamma>0
\end{array}\right.
$$

$i$ крім того, існуе $K_{0} \in(0,+\infty)$ таке, що

$$
\|x(t, \varepsilon, \theta)\|_{F\left(m ; \varepsilon_{0} ; \theta\right)} \leq K_{0}\|u(t, \varepsilon, \theta)\|_{F\left(m ; \varepsilon_{0} ; \theta\right)} .
$$

Доведення леми наведено в роботі [6].

Лема 2. Нехай рівняння (2) таке, що існують матрииі $L_{1}(t, \varepsilon), L_{2}(t, \varepsilon) \in$ $\in S_{2}\left(m ; \varepsilon_{0}\right) \operatorname{maki}, щ_{0}$

a) $\left|\operatorname{det} L_{k}(t, \varepsilon)\right| \geq a_{0}>0,(k=1,2)$,

б) $L_{1}^{-1}(t, \varepsilon) A(t, \varepsilon) L_{1}(t, \varepsilon)=D_{1}(t, \varepsilon)=\left(d_{j k}^{1}(t, \varepsilon)\right)_{j, k=\overline{1, N}}$,

$$
L_{2}(t, \varepsilon) B(t, \varepsilon) L_{2}^{-1}(t, \varepsilon)=D_{2}(t, \varepsilon)=\left(d_{j k}^{2}(t, \varepsilon)\right)_{j, k=\overline{1, N}}
$$

де $D_{1}(t, \varepsilon), D_{2}(t, \varepsilon)$ - нижні трикутні матриці $N$-го порядку, що належать до $\kappa л a c y S_{2}\left(m ; \varepsilon_{0}\right) ; d_{j j}^{1}(t, \varepsilon)=\lambda_{j}^{1}(t, \varepsilon), d_{k k}^{2}(t, \varepsilon)=\lambda_{k}^{2}(t, \varepsilon)$.

Тоді підстановкою

$$
X=L_{1}(t, \varepsilon) Y L_{2}(t, \varepsilon)
$$

рівняння (2) зводитвся до вигляду:

$$
\begin{aligned}
\frac{d Y}{d t}=D_{1}(t, \varepsilon) & Y-Y D_{2}(t, \varepsilon)-\varepsilon H_{1}(t, \varepsilon) Y-\varepsilon Y H_{2}(t, \varepsilon)+ \\
& +F_{1}(t, \varepsilon, \theta)+\mu \Phi_{1}(t, \varepsilon, \theta, Y)
\end{aligned}
$$

$\partial e$

$$
\begin{gathered}
H_{1}(t, \varepsilon)=\frac{1}{\varepsilon} L_{1}^{-1}(t, \varepsilon) \frac{d L_{1}(t, \varepsilon)}{d t}, \quad H_{2}(t, \varepsilon)=\frac{1}{\varepsilon} \frac{d L_{2}(t, \varepsilon)}{d t} L_{2}^{-1}(t, \varepsilon) \\
F_{1}(t, \varepsilon, \theta)=L_{1}^{-1}(t, \varepsilon) F(t, \varepsilon, \theta) L_{2}^{-1}(t, \varepsilon) \\
\Phi_{1}(t, \varepsilon, \theta, Y)=L_{1}^{-1}(t, \varepsilon) \Phi\left(t, \varepsilon, \theta, L_{1}(t, \varepsilon) Y L_{2}(t, \varepsilon)\right) L_{2}^{-1}(t, \varepsilon) .
\end{gathered}
$$

Наук. вісник Ужгород. ун-ту. 2021. Том 39, № 2 ISSN 2616-7700 (print), 2708-9568 (online) 
Доведення. Щоб переконатися в справедливості леми, достатньо в рівнянні (2) зробити підстановку (6) та використати умови леми.

Лема 3. Нехай задано лінійне матричне рівняння

$$
\frac{d X}{d t}=\left(D_{1}(t, \varepsilon)+\sum_{l=1}^{q} B_{1 l}(t, \varepsilon, \theta) \mu^{l}\right) X-X\left(D_{2}(t, \varepsilon)+\sum_{l=1}^{q} B_{2 l}(t, \varepsilon, \theta) \mu^{l}\right)
$$

$D_{1}(t, \varepsilon), D_{2}(t, \varepsilon)$ - тіж самі, що й в лемі 2, $B_{1 l}(t, \varepsilon, \theta), B_{2 l}(t, \varepsilon, \theta)(l=\overline{1, q})$ належать до класу $F_{2}\left(m ; \varepsilon_{0} ; \theta\right), \mu \in(0,1)$ - малий дійсний параметр.

Тоді для достатнъо малих значень н існуе перетворення

$$
X=\left(E+\sum_{l=1}^{q} Q_{1 l}(t, \varepsilon, \theta) \mu^{l}\right) Y\left(E+\sum_{l=1}^{q} Q_{2 l}(t, \varepsilon, \theta) \mu^{l}\right),
$$

де $Q_{1 l}(t, \varepsilon, \theta), Q_{2 l}(t, \varepsilon, \theta)(l=\overline{1, q})$ належать до класу $F_{2}\left(m ; \varepsilon_{0} ; \theta\right)$, що приводить рівняння (8) до вигляду

$$
\begin{aligned}
& \frac{d Y}{d t}=\left(D_{1}(t, \varepsilon)+\sum_{l=1}^{q} U_{1 l}(t, \varepsilon) \mu^{l}+\varepsilon \sum_{l=1}^{q} V_{1 l}(t, \varepsilon, \theta) \mu^{l}+\mu^{q+1} W_{1}(t, \varepsilon, \theta, \mu)\right) Y- \\
& -Y\left(D_{2}(t, \varepsilon)+\sum_{l=1}^{q} U_{2 l}(t, \varepsilon) \mu^{l}+\varepsilon \sum_{l=1}^{q} V_{2 l}(t, \varepsilon, \theta) \mu^{l}+\mu^{q+1} W_{2}(t, \varepsilon, \theta, \mu)\right),
\end{aligned}
$$

де $U_{1 l}(t, \varepsilon), U_{2 l}(t, \varepsilon)(l=\overline{1, q})$ - діагональні матриці, що належать до класу $S_{2}\left(m ; \varepsilon_{0}\right), V_{1 l}, V_{2 l}, W_{1}, W_{2}(l=\overline{1, q})$ - квадратні матрищі, що належать до класу $F\left(m-1 ; \varepsilon_{0} ; \theta\right)$.

Доведення. Підставимо вираз (9) в рівняння (8) і вимагатимемо, щоб перетворене рівняння мало вигляд (10). Тоді одержимо наступні матричні рівняння для визначення матриць $Q_{11} \ldots Q_{1 q} Q_{21} \ldots Q_{2 q}$ :

$$
\begin{aligned}
& \frac{d Q_{11}}{d t}=D_{1}(t, \varepsilon) Q_{11}-Q_{11} D_{1}(t, \varepsilon)+B_{11}(t, \varepsilon, \theta)-U_{11}(t, \varepsilon)-\varepsilon V_{11}(t, \varepsilon, \theta), \\
& \frac{d Q_{1 s}}{d t}=D_{1}(t, \varepsilon) Q_{1 s}-Q_{1 s} D_{1}(t, \varepsilon)+B_{1 s}(t, \varepsilon, \theta)+\sum_{\nu=1}^{s-1} B_{1 \nu}(t, \varepsilon, \theta) Q_{1, s-\nu}- \\
& -\sum_{\nu=1}^{s-1} Q_{1 \nu} U_{1, s-\nu}-\varepsilon \sum_{\nu=1}^{s-1} Q_{1 \nu} V_{1, s-\nu}-U_{1 s}(t, \varepsilon)-\varepsilon V_{1 s}(t, \varepsilon, \theta), s=\overline{2, q}, \\
& \frac{d Q_{21}}{d t}=D_{2}(t, \varepsilon) Q_{21}-Q_{21} D_{2}(t, \varepsilon)-B_{21}(t, \varepsilon, \theta)+U_{21}(t, \varepsilon)+\varepsilon V_{21}(t, \varepsilon, \theta), \\
& \frac{d Q_{2 s}}{d t}=D_{2}(t, \varepsilon) Q_{2 s}-Q_{2 s} D_{2}(t, \varepsilon)-B_{2 s}(t, \varepsilon, \theta)-\sum_{\nu=1}^{s-1} B_{2 \nu}(t, \varepsilon, \theta) Q_{2, s-\nu}+ \\
& +\sum_{\nu=1}^{s-1} Q_{2 \nu} U_{2, s-\nu}+\varepsilon \sum_{\nu=1}^{s-1} Q_{2 \nu} V_{2, s-\nu}+U_{2 s}(t, \varepsilon)+\varepsilon V_{2 s}(t, \varepsilon, \theta), s=\overline{2, q} .
\end{aligned}
$$

Розділ 1: Математика і статистика 
Матриці $W_{1}(t, \varepsilon, \theta, \mu), W_{2}(t, \varepsilon, \theta, \mu)$ визначаться з співвідношень:

$$
\begin{aligned}
\left(E+\sum_{l=1}^{q} Q_{1 l} \mu^{l}\right) & W_{1}=\sum_{s=0}^{q-1}\left[\sum_{\sigma+\delta=s+q+1}\left(B_{1 \sigma} Q_{1 \sigma}-Q_{1 \sigma} U_{1 \sigma}\right)\right] \mu^{s}- \\
& -\varepsilon \sum_{s=0}^{q-1}\left[\sum_{\sigma+\delta=s+q+1} Q_{1 \sigma} V_{1 \delta}\right] \mu^{s}, \\
\left(E+\sum_{l=1}^{q} Q_{2 l} \mu^{l}\right) & W_{2}=-\sum_{s=0}^{q-1}\left[\sum_{\sigma+\delta=s+q+1}\left(B_{2 \sigma} Q_{2 \sigma}-Q_{2 \sigma} U_{2 \sigma}\right)\right] \mu^{s}+ \\
& +\varepsilon \sum_{s=0}^{q-1}\left[\sum_{\sigma+\delta=s+q+1} Q_{2 \sigma} V_{2 \delta}\right] \mu^{s} .
\end{aligned}
$$

$$
\begin{aligned}
& \text { Покладемо } Q_{1 s}=\left(q_{j k}^{1 s}\right)_{j, k=\overline{1, N}}, Q_{2 s}=\left(q_{j k}^{2 s}\right)_{j, k=\overline{1, N}}, \\
& B_{1 s}(t, \varepsilon, \theta)=\left(b_{j k}^{1 s}(t, \varepsilon, \theta)\right)_{j, k=\overline{1, N}}, B_{2 s}(t, \varepsilon, \theta)=\left(b_{j k}^{2 s}(t, \varepsilon, \theta)\right)_{j, k=\overline{1, N}}, \\
& U_{1 s}(t, \varepsilon)=\left(u_{j k}^{1 s}(t, \varepsilon)\right)_{j, k=\overline{1, N}}, U_{2 s}(t, \varepsilon)=\left(u_{j k}^{2 s}(t, \varepsilon)\right)_{j, k=\overline{1, N}}, \\
& V_{1 s}(t, \varepsilon, \theta)=\left(v_{j k}^{1 s}(t, \varepsilon, \theta)\right)_{j, k=\overline{1, N}}, V_{2 s}(t, \varepsilon, \theta)=\left(v_{j k}^{2 s}(t, \varepsilon, \theta)\right)_{j, k=\overline{1, N}}, \mathrm{~s}=1,2, \\
& \text { і запишемо системи }(11)-(14) \text { в компонентній формі: }
\end{aligned}
$$

$$
\frac{d q_{j k}^{11}}{d t}=\sum_{\nu=1}^{j} d_{j \nu}^{1}(t, \varepsilon) q_{\nu k}^{11}-\sum_{\nu=k}^{N} d_{\nu k}^{1}(t, \varepsilon) q_{j \nu}^{11}+b_{j k}^{11}(t, \varepsilon, \theta)-u_{j k}^{11}(t, \varepsilon)-\varepsilon v_{j k}^{11}(t, \varepsilon, \theta)
$$

$j, k=\overline{1, N}$

$$
\begin{gathered}
\frac{d q_{j k}^{1 s}}{d t}=\sum_{\nu=1}^{j} d_{j \nu}^{1}(t, \varepsilon) q_{\nu k}^{1 s}-\sum_{\nu=k}^{N} d_{\nu k}^{1}(t, \varepsilon) q_{j \nu}^{1 s}+b_{j k}^{1 s}(t, \varepsilon, \theta)+ \\
+\left(\sum_{\nu=1}^{s-1} B_{1 \nu}(t, \varepsilon, \theta) Q_{1, s-\nu}\right)_{j k}-\left(\sum_{\nu=1}^{s-1} Q_{1 \nu} U_{1, s-\nu}(t, \varepsilon)\right)_{j k}-\varepsilon\left(\sum_{\nu=1}^{s-1} Q_{1 \nu} V_{1, s-\nu}(t, \varepsilon, \theta)\right)_{j k}- \\
-u_{j k}^{1 s}(t, \varepsilon)-\varepsilon v_{j k}^{1 s}(t, \varepsilon, \theta), s=\overline{2, q}, j, k=\overline{1, N} \\
\frac{d q_{j k}^{21}}{d t}=\sum_{\nu=1}^{j} d_{j \nu}^{2}(t, \varepsilon) q_{\nu k}^{21}-\sum_{\nu=k}^{N} d_{\nu k}^{2}(t, \varepsilon) q_{j \nu}^{21}-b_{j k}^{21}(t, \varepsilon, \theta)+u_{j k}^{21}(t, \varepsilon)+\varepsilon v_{j k}^{21}(t, \varepsilon, \theta), \\
j, k=19) \\
\frac{d q_{j k}^{2 s}}{d t}=\sum_{\nu=1}^{j} d_{j \nu}^{2}(t, \varepsilon) q_{\nu k}^{2 s}-\sum_{\nu=k}^{N} d_{\nu k}^{2}(t, \varepsilon) q_{j \nu}^{2 s}-b_{j k}^{2 s}(t, \varepsilon, \theta)- \\
-\left(\sum_{\nu=1}^{s-1} B_{2 \nu}(t, \varepsilon, \theta) Q_{2, s-\nu}\right)_{j k}+\left(\sum_{\nu=1}^{s-1} Q_{2 \nu} U_{2, s-\nu}(t, \varepsilon)\right)_{j k}+\varepsilon\left(\sum_{\nu=1}^{s-1} Q_{2 \nu} V_{2, s-\nu}(t, \varepsilon, \theta)\right)_{j k}
\end{gathered}
$$

Наук. вісник Ужгород. ун-ту. 2021. Том 39, № 2 ISSN 2616-7700 (print), 2708-9568 (online) 


$$
+u_{j k}^{2 s}(t, \varepsilon)+\varepsilon v_{j k}^{2 s}(t, \varepsilon, \theta), s=\overline{2, q}, j, k=\overline{1, N} .
$$

Розглянемо рівняння (17). Запишемо його детальніше:

$$
\begin{aligned}
& \frac{d q_{1 N}^{11}}{d t}=\left(d_{11}^{1}(t, \varepsilon)-d_{N N}^{1}(t, \varepsilon)\right) q_{1 N}^{11}+b_{1 N}^{11}(t, \varepsilon, \theta)-u_{1 N}^{11}(t, \varepsilon)-\varepsilon v_{1 N}^{11}(t, \varepsilon, \theta), \\
& \frac{d q_{11}^{11}}{d t}=-\sum_{\nu=2}^{N} d_{\nu 1}^{1}(t, \varepsilon) q_{1 \nu}^{11}+b_{11}^{11}(t, \varepsilon, \theta)-u_{11}^{11}(t, \varepsilon)-\varepsilon v_{11}^{11}(t, \varepsilon, \theta), \\
& \frac{d q_{2 N}^{11}}{d t}=\left(d_{22}^{1}(t, \varepsilon)-d_{N N}^{1}(t, \varepsilon)\right) q_{2 N}^{11}+d_{2 N}^{1}(t, \varepsilon) q_{1 N}^{11}+b_{2 N}^{11}(t, \varepsilon, \theta)-u_{2 N}^{11}(t, \varepsilon)-\varepsilon v_{2 N}^{11}(t, \varepsilon, \theta) \text {, } \\
& \frac{d q_{21}^{11}}{d t}=\left(d_{22}^{1}(t, \varepsilon)-d_{11}^{1}(t, \varepsilon)\right) q_{21}^{11}+d_{21}^{1}(t, \varepsilon) q_{11}^{11}- \\
& -\sum_{\nu=2}^{N} d_{\nu 1}^{1}(t, \varepsilon) q_{2 \nu}^{11}+b_{21}^{11}(t, \varepsilon, \theta)-u_{21}^{11}(t, \varepsilon)-\varepsilon v_{21}^{11}(t, \varepsilon, \theta) \\
& \frac{d q_{N N}^{11}}{d t}=\sum_{\nu=1}^{N-1} d_{N \nu}^{1}(t, \varepsilon) q_{\nu N}^{11}+b_{N N}^{11}-u_{N N}^{11}(t, \varepsilon)-\varepsilon v_{N N}^{11}(t, \varepsilon, \theta), \\
& \frac{d q_{N 1}^{11}}{d t}=\left(d_{N N}^{1}(t, \varepsilon)-d_{11}^{1}(t, \varepsilon)\right) q_{N 1}^{11}+\sum_{\nu=1}^{N-1} d_{N \nu}^{1}(t, \varepsilon) q_{\nu 1}^{11}-\sum_{\nu=2}^{N} d_{\nu 1}^{1}(t, \varepsilon) q_{N \nu}^{11}+ \\
& +b_{N 1}^{11}(t, \varepsilon, \theta)-u_{N 1}^{11}(t, \varepsilon)-\varepsilon v_{N 1}^{11}(t, \varepsilon, \theta) .
\end{aligned}
$$

Розглянемо рівняння (21). Нехай

$$
b_{j k}^{11}(t, \varepsilon, \theta)=\sum_{n=-\infty}^{\infty} b_{j k, n}^{11}(t, \varepsilon) \exp (i n \theta(t, \varepsilon)), j, k=\overline{1, N} .
$$

Покладемо

$$
q_{j k}^{11}(t, \varepsilon, \theta)=\sum_{n=-\infty}^{\infty} q_{j k, n}^{11}(t, \varepsilon) \exp (i n \theta(t, \varepsilon)), j, k=\overline{1, N}
$$

Тоді

$$
\begin{gathered}
q_{1 N}^{11}(t, \varepsilon, \theta)=-\sum_{n=-\infty}^{\infty} \frac{b_{1 N, n}^{11}(t, \varepsilon)}{d_{11}^{1}(t, \varepsilon)-d_{N N}^{1}(t, \varepsilon)-i n \varphi(t, \varepsilon)} \exp (i n \theta(t, \varepsilon)), \\
v_{1 N}^{11}(t, \varepsilon, \theta)=\frac{1}{\varepsilon} \sum_{n=-\infty}^{\infty} \frac{u_{1 N}^{11}(t, \varepsilon)=0}{d t}\left(\frac{b_{1 N, n}^{11}(t, \varepsilon)}{d_{11}^{1}(t, \varepsilon)-d_{N N}^{1}(t, \varepsilon)-i n \varphi(t, \varepsilon)}\right) \exp (i n \theta(t, \varepsilon)) .
\end{gathered}
$$

Розділ 1: Математика і статистика 
Аналогічно

$$
\begin{gathered}
q_{1 N}^{21}(t, \varepsilon, \theta)=\sum_{n=-\infty}^{\infty} \frac{b_{1 N, n}^{21}(t, \varepsilon)}{d_{11}^{2}(t, \varepsilon)-d_{N N}^{2}(t, \varepsilon)-i n \varphi(t, \varepsilon)} \exp (i n \theta(t, \varepsilon)), \\
v_{1 N}^{21}(t, \varepsilon, \theta)=-\frac{1}{\varepsilon} \sum_{n=-\infty}^{\infty} \frac{u_{1 N}^{21}(t, \varepsilon)=0,}{d t}\left(\frac{b_{1 N, n}^{21}(t, \varepsilon)}{d_{11}^{2}(t, \varepsilon)-d_{N N}^{2}(t, \varepsilon)-i n \varphi(t, \varepsilon)}\right) \exp (\operatorname{in} \theta(t, \varepsilon)) .
\end{gathered}
$$

Внаслідок умови $1^{0}$ маємо:

$q_{1 n}^{11}(t, \varepsilon, \theta), q_{1 n}^{21}(t, \varepsilon, \theta) \in F\left(m ; \varepsilon_{0} ; \theta\right), v_{1 n}^{11}(t, \varepsilon, \theta), v_{1 n}^{21}(t, \varepsilon, \theta) \in F\left(m-1 ; \varepsilon_{0} ; \theta\right)$.

Аналогічно отримуємо: $q_{1, N-1}^{11}, \ldots, q_{12}^{11}, q_{1, N-1}^{21}, \ldots, q_{12}^{21} \in F\left(m ; \varepsilon_{0} ; \theta\right)$, $v_{1, N-1}^{11}, \ldots, v_{12}^{11}, v_{1, N-1}^{21}, \ldots, v_{12}^{21} \in F\left(m-1 ; \varepsilon_{0} ; \theta\right)$.

Далі розглянемо рівняння (22). Функція

$$
c_{11}^{11}=-\sum_{\nu=2}^{N} d_{\nu 1}^{1}(t, \varepsilon) q_{1 \nu}^{11}+b_{11}^{11}(t, \varepsilon, \theta),
$$

очевидно, належить до класу $F\left(m ; \varepsilon_{0} ; \theta\right)$. Покладемо:

$$
\begin{gathered}
u_{11}^{11}(t, \varepsilon)=\Gamma_{0}\left[c_{11}^{11}(t, \varepsilon, \theta)\right], \\
q_{11}^{11}(t, \varepsilon, \theta)=\sum_{\substack{n=-\infty \\
(n \neq 0)}}^{\infty} \frac{\Gamma_{n}\left[c_{11}^{11}(t, \varepsilon, \theta)\right]}{i n \varphi(t, \varepsilon)} \exp (i n \theta(t, \varepsilon)), \\
v_{11}^{11}(t, \varepsilon, \theta)=-\frac{1}{\varepsilon} \sum_{\substack{n=-\infty \\
(n \neq 0)}}^{\infty} \frac{d}{d t}\left(\frac{\Gamma_{n}\left[c_{11}^{11}(t, \varepsilon, \theta)\right]}{i n \varphi(t, \varepsilon)}\right) \exp (i n \theta(t, \varepsilon)) .
\end{gathered}
$$

Аналогічно

$$
\begin{gathered}
u_{11}^{21}(t, \varepsilon)=\Gamma_{0}\left[c_{11}^{21}(t, \varepsilon, \theta)\right], \\
q_{11}^{21}(t, \varepsilon, \theta)=\sum_{\substack{n=-\infty \\
(n \neq 0)}}^{\infty} \frac{\Gamma_{n}\left[c_{11}^{21}(t, \varepsilon, \theta)\right]}{i n \varphi(t, \varepsilon)} \exp (\operatorname{in} \theta(t, \varepsilon)), \\
v_{11}^{21}(t, \varepsilon, \theta)=-\frac{1}{\varepsilon} \sum_{\substack{n=-\infty \\
(n \neq 0)}}^{\infty} \frac{d}{d t}\left(\frac{\Gamma_{n}\left[c_{11}^{21}(t, \varepsilon, \theta)\right]}{i n \varphi(t, \varepsilon)}\right) \exp (i n \theta(t, \varepsilon)),
\end{gathered}
$$

де

$$
c_{11}^{21}=-\sum_{\nu=2}^{N} d_{\nu 1}^{2}(t, \varepsilon) q_{1 \nu}^{21}-b_{11}^{21}(t, \varepsilon, \theta) .
$$

Очевидно, що $q_{11}^{11}(t, \varepsilon, \theta), q_{11}^{21}(t, \varepsilon, \theta)$ належать до класу $F\left(m ; \varepsilon_{0} ; \theta\right), u_{11}^{11}(t, \varepsilon)$, $u_{11}^{21}(t, \varepsilon)$ належать до класу $S(m ; \varepsilon), v_{11}^{11}(t, \varepsilon, \theta), v_{11}^{21}(t, \varepsilon, \theta)$ належать до класу $F\left(m-1 ; \varepsilon_{0} ; \theta\right)$.

Наук. вісник Ужгород. ун-ту. 2021. Том 39, № 2 ISSN 2616-7700 (print), 2708-9568 (online) 
Аналогічно показуємо, що функції $u_{22}^{11}(t, \varepsilon), u_{22}^{21}(t, \varepsilon), \ldots, u_{N N}^{11}(t, \varepsilon), u_{N N}^{21}(t, \varepsilon)$ належать до класу

$S\left(m ; \varepsilon_{0}\right), q_{j k}^{11}(t, \varepsilon, \theta), q_{j k}^{21}(t, \varepsilon, \theta) \in F\left(m ; \varepsilon_{0} ; \theta\right) v_{j k}^{11}(t, \varepsilon, \theta), v_{j k}^{21}(t, \varepsilon, \theta) \in F\left(m-1 ; \varepsilon_{0} ; \theta\right)(j \neq k)$.

Таким чином, матриці $U_{11}(t, \varepsilon), \ldots, U_{1 q}(t, \varepsilon), U_{21}(t, \varepsilon), \ldots, U_{2 q}(t, \varepsilon)$ - діагональні з елементами, що належать до класу $S(m ; \varepsilon)$. Матриці $W_{1}, W_{2}$ визначаються для достатньо малих $\mu$ з рівнянь (15), (16).

Лему доведено.

Припустимо, що невідома матриця $Y$ у рівнянні (7) належить деякій замкненій і обмеженій області $D^{*} \subset \mathbb{C}^{N \times N}$.

Лема 4. Нехай матриия-функиія $\Phi_{1}(t, \varepsilon, \theta, Y)$ у рівнянні (7) має в $D^{*}$ неперервні похідні за $Y$ в сенсі Фреше [9] до порядку $2 q+1$ включно, $i$ якщо $Y \in F_{2}\left(m ; \varepsilon_{0} ; \theta\right)$, то иі похідні також з класу $F_{2}\left(m ; \varepsilon_{0} ; \theta\right)$. Тоді існуе таке $\mu_{0} \in(0,1)$, що для всіх $\mu_{1} \in\left(0, \mu_{0}\right)$ існуе перетворення

$$
Y=\Psi_{1}(t, \varepsilon, \theta, \mu)+\Psi_{2}(t, \varepsilon, \theta, \mu) Z \Psi_{3}(t, \varepsilon, \theta, \mu),
$$

де $Z \in D^{* *} \subset \mathbb{C}^{N \times N}, \Psi_{1}(t, \varepsilon, \theta, \mu), \Psi_{2}(t, \varepsilon, \theta, \mu), \Psi_{3}(t, \varepsilon, \theta, \mu) \in F_{2}\left(m ; \varepsilon_{0} ; \theta\right), u_{0}$ зводить рівняння (7) до вигляду:

$$
\begin{aligned}
& \frac{d Z}{d t}=\left(D_{1}(t, \varepsilon)+\sum_{l=1}^{q} U_{1 l}(t, \varepsilon) \mu^{l}\right) Z-Z\left(D_{2}(t, \varepsilon)+\sum_{l=1}^{q} U_{2 l}(t, \varepsilon) \mu^{l}\right)+ \\
&+\varepsilon K(t, \varepsilon, \theta, \mu)+\mu^{2 q} C(t, \varepsilon, \theta, \mu)+\varepsilon V_{1}(t, \varepsilon, \theta, \mu) Z-\varepsilon Z V_{2}(t, \varepsilon, \theta, \mu)+ \\
& \quad+\mu^{q+1}\left(R_{1}(t, \varepsilon, \theta, \mu) Z-Z R_{2}(t, \varepsilon, \theta, \mu)\right)+\mu \Phi_{2}(t, \varepsilon, \theta, Z, \mu),
\end{aligned}
$$

де $K \in F_{2}\left(m-1 ; \varepsilon_{0} ; \theta\right), U_{1 l}, U_{2 l} \in S_{2}\left(m ; \varepsilon_{0}\right), R_{1}, R_{2}, C \in F_{2}\left(m ; \varepsilon_{0} ; \theta\right), V_{1}, V_{2} \in$ $\in F\left(m-1 ; \varepsilon_{0} ; \theta\right)$, матрии,я-функція $\Phi_{2}$ належить до класу $F_{2}\left(m ; \varepsilon_{0} ; \theta\right)$ відносно $t, \varepsilon, \theta$, неперервно диферениійовна в сенсі Фреше за $Z$ i містить доданки не нижче другого порядку відносно $Z$.

Доведення. Поряд з рівнянням (7) розглянемо допоміжне рівняння:

$$
\varphi(t, \varepsilon) \frac{d \Xi}{d \theta}=D_{1}(t, \varepsilon) \Xi-\Xi D_{2}(t, \varepsilon)+F_{1}(t, \varepsilon, \theta)+\mu \Phi_{1}(t, \varepsilon, \theta, \Xi),
$$

де $t, \varepsilon, \varphi$ розглядаються як сталі.

Оскільки матриці-функції $F_{1}, \Phi_{1} \in 2 \pi$-періодичними функціями змінної $\theta$, то для знаходження $2 \pi$-періодичного по $\theta$ розв'язку рівняння (25) можна застосувати метод малого параметра Пуанкаре [10], згідно якому у випадку аналітичності відносно $\Xi$ нелінійності $\Phi_{1}(t, \varepsilon, \theta, \Xi)$ цей розв’язок шукається у вигляді ряду за степенями малого параметру $\mu$. У випадку виконання умови леми наближений $2 \pi$-періодичний по $\theta$ розв'язок рівняння (25) можна шукати у вигляді часткової суми цього ряду:

$$
\Xi(t, \varepsilon, \theta, \mu)=\sum_{k=0}^{2 q-1} \Xi_{k}(t, \varepsilon, \theta) \mu^{k} .
$$

Розділ 1: Математика і статистика 
При цьому матриця-функція $\Xi_{0}(t, \varepsilon, \theta)$ визначиться 3 рівняння:

$$
\varphi(t, \varepsilon) \frac{d \Xi_{0}}{d \theta}=D_{1}(t, \varepsilon) \Xi_{0}-\Xi_{0} D_{2}(t, \varepsilon)+F_{1}(t, \varepsilon, \theta) .
$$

Згідно з термінологією теорії коливань назвемо це рівняння породжуючим.

Нехай

$$
\Xi_{0}=\left(\xi_{j k}^{0}(t)\right)_{j, k=\overline{1, N}}, F_{1}(t, \varepsilon, \theta)=\left(f_{j k}^{1}(t, \varepsilon, \theta)\right)_{j, k=\overline{1, N}} .
$$

Тоді, розписуючи рівняння (27) у компонентній формі, прийдемо до скалярної лінійної системи диференціальних рівнянь вигляду:

$$
\varphi(t, \varepsilon) \frac{d \xi_{j k}^{0}}{d \theta}=\sum_{s=1}^{j} d_{j s}^{1}(t, \varepsilon) \xi_{s k}^{0}-\sum_{s=k}^{N} d_{s k}^{2}(t, \varepsilon) \xi_{j s}^{0}+f_{j k}^{1}(t, \varepsilon, \theta), j, k=\overline{1, N} .
$$

Або

$$
\begin{gathered}
\varphi(t, \varepsilon) \frac{d \xi_{1 N}^{0}}{d \theta}=\left(d_{11}^{1}(t, \varepsilon)-d_{N N}^{2}(t, \varepsilon)\right) \xi_{1 N}^{0}+f_{1 N}^{1}(t, \varepsilon, \theta), \\
\ldots \\
\varphi(t, \varepsilon) \frac{d \xi_{11}^{0}}{d \theta}=\left(d_{11}^{1}(t, \varepsilon)-d_{11}^{2}(t, \varepsilon)\right) \xi_{11}^{0}-\sum_{s=2}^{N} d_{s 1}^{2}(t, \varepsilon) \xi_{1 s}^{0}+f_{11}^{1}(t, \varepsilon, \theta), \\
\varphi(t, \varepsilon) \frac{d \xi_{2 N}^{0}}{d \theta}=\left(d_{22}^{1}(t, \varepsilon)-d_{N N}^{2}(t, \varepsilon)\right) \xi_{2 N}^{0}+d_{21}^{1}(t, \varepsilon) \xi_{1 n}^{0}+f_{2 N}^{1}(t, \varepsilon, \theta), \\
\ldots \\
\varphi(t, \varepsilon) \frac{d \xi_{21}^{0}}{d \theta}=\left(d_{22}^{1}(t, \varepsilon)-d_{11}^{2}(t, \varepsilon)\right) \xi_{21}^{0}+d_{21}^{1}(t, \varepsilon) \xi_{11}^{0}-\sum_{s=2}^{N} d_{s 1}^{2}(t, \varepsilon) \xi_{2 s}^{0}+f_{21}^{1}(t, \varepsilon, \theta), \\
\ldots \\
\varphi(t, \varepsilon) \frac{d \xi_{N N}^{0}}{d \theta}=\left(d_{N N}^{1}(t, \varepsilon)-d_{N N}^{2}(t, \varepsilon)\right) \xi_{N N}^{0}+\sum_{s=1}^{N-1} d_{N s}^{1}(t, \varepsilon) \xi_{s N}^{0}+f_{N N}^{1}(t, \varepsilon, \theta), \\
\ldots \\
\varphi(t, \varepsilon) \frac{d \xi_{N 1}^{0}}{d \theta}=\left(d_{N N}^{1}(t, \varepsilon)-d_{11}^{2}(t, \varepsilon)\right) \xi_{N 1}^{0}+\sum_{s=1}^{N-1} d_{N s}^{1}(t, \varepsilon) \xi_{s 1}^{0}-\sum_{s=2}^{N} d_{s 1}^{2}(t, \varepsilon) \xi_{N s}^{0}+f_{N 1}^{1}(t, \varepsilon, \theta) .
\end{gathered}
$$

Розглянемо перше з рівнянь цієї системи. Розкладемо функцію $f_{1 N}^{1}(t, \varepsilon, \theta)$ в абсолютно та рівномірно збіжний ряд Фур'є

$$
f_{1 N}^{1}(t, \varepsilon, \theta)=\sum_{n=-\infty}^{\infty} f_{1 N, n}^{1}(t, \varepsilon) \exp (i n \theta)
$$

і шукатимемо функцію $\xi_{1 N}^{0}$ у вигляді аналогічного ряду

$$
\xi_{1 N}^{0}(t, \varepsilon, \theta)=\sum_{n=-\infty}^{\infty} \xi_{1 N, n}^{1}(t, \varepsilon) \exp (i n \theta)
$$

Тоді відносно $\xi_{1 N, n}^{1}(t, \varepsilon)$ дістанемо рівняння

$$
\left(d_{11}^{1}(t, \varepsilon)-d_{N N}^{2}(t, \varepsilon)-i n \varphi(t, \varepsilon)\right) \xi_{1 N, n}^{0}=-f_{1 N, n}^{1}(t, \varepsilon), n \in \mathbb{Z}
$$

Наук. вісник Ужгород. ун-ту. 2021. Том 39, № 2 ISSN 2616-7700 (print), 2708-9568 (online) 
Внаслідок умови $2^{0}$ функція $\xi_{1 N}^{0}(t, \varepsilon, \theta) \in$ функцією класу $F\left(m ; \varepsilon_{0} ; \theta\right)$. Аналогічно і решта функцій $\xi_{j k}^{0}(t, \varepsilon, \theta)(j, k=\overline{1, N})$ внаслідок тієї ж умови $2^{0} є$ функціями класу $F\left(m ; \varepsilon_{0} ; \theta\right)$, а це означає, що матриця-функція $\Xi_{0}(t, \varepsilon, \theta)$ належить до класу $F_{2}\left(m ; \varepsilon_{0} ; \theta\right)$.

Запишемо тепер часткову суму ряду Тейлора матриці-функції $\Phi_{1}(t, \varepsilon, \theta, \Xi)$ :

$$
\Phi_{1}^{2 q-1}=\sum_{k=0}^{2 q-1} \frac{\Phi_{1}^{(k)}\left(t, \varepsilon, \theta, \Xi_{0}\right)}{k !}\left(\Xi-\Xi_{0}\right)^{k} .
$$

Тоді коефіцієнти $\Xi_{k}(t, \varepsilon, \theta)(k=\overline{1,2 q-1})$ суми $(26)$ визначаться як $2 \pi$-періодичні за $\theta$ розв'язки ланцюжка рівнянь:

$$
\begin{gathered}
\varphi(t, \varepsilon) \frac{d \Xi_{0}}{d \theta}=D_{1}(t, \varepsilon) \Xi_{0}-\Xi_{0} D_{2}(t, \varepsilon)+F_{1}(t, \varepsilon, \theta) \\
\varphi(t, \varepsilon) \frac{d \Xi_{1}}{d \theta}=D_{1}(t, \varepsilon) \Xi_{1}-\Xi_{1} D_{2}(t, \varepsilon)+\Phi_{1}\left(t, \varepsilon, \theta, \Xi_{0}\right), \\
\varphi(t, \varepsilon) \frac{d \Xi_{2}}{d \theta}=D_{1}(t, \varepsilon) \Xi_{2}-\Xi_{2} D_{2}(t, \varepsilon)+\frac{d \Phi_{1}\left(t, \varepsilon, \theta, \Xi_{0}\right)}{d \theta} \Xi_{1}, \\
\varphi(t, \varepsilon) \frac{d \Xi_{3}}{d \theta}=D_{1}(t, \varepsilon) \Xi_{3}-\Xi_{3} D_{2}(t, \varepsilon)+\frac{d \Phi_{1}\left(t, \varepsilon, \theta, \Xi_{0}\right)}{d \theta} \Xi_{2}+\frac{1}{2} \frac{d^{2} \Phi_{1}\left(t, \varepsilon, \theta, \Xi_{0}\right)}{d \theta^{2}} \Xi_{1}^{2}, \\
\varphi(t, \varepsilon) \frac{d \Xi_{s}}{d \theta}=D_{1}(t, \varepsilon) \Xi_{s}-\Xi_{s} D_{2}(t, \varepsilon)+\frac{d \Phi_{1}\left(t, \varepsilon, \theta, \Xi_{0}\right)}{d \theta} \Xi_{s-1}+ \\
+P_{s}\left(t, \varepsilon, \theta, \Xi_{0}, \cdots, \Xi_{s-2}\right), s=\frac{\pi, 2 q-1}{4,}
\end{gathered}
$$

де $P_{s}$ - матричні поліноми відносно $\Xi_{0}, \cdots, \Xi_{s-2}$ з коефіцієнтами, що належать до класу $F_{2}\left(m ; \varepsilon_{0} ; \theta\right)$.

Рівняння (30) - породжуюче рівняння, і наявність у нього розв'язку класу $F_{2}\left(m ; \varepsilon_{0} ; \theta\right)$ встановлено вище. За властивостями функції $\Phi_{1}$ вільний член $\Phi\left(t, \varepsilon, \theta, \Xi_{0}\right)$ рівняння (31) також є функцією класу $F_{2}\left(m ; \varepsilon_{0} ; \theta\right)$. I, отже, функція $\Xi_{1}(t, \varepsilon, \theta)$ є функцією класу $F_{2}\left(m ; \varepsilon_{0} ; \theta\right)$. Аналогічно і всі функції $\Xi_{2}, \cdots, \Xi_{s}$ теж $\epsilon$ функціями класу $F_{2}\left(m ; \varepsilon_{0} ; \theta\right)$. Отже, функція $(26) \in$ функцією того ж класу.

Здійснимо у рівнянні (7) підстановку

$$
Y=\Xi(t, \varepsilon, \theta, \mu)+Z_{1},
$$

де $Z_{1}$ - нова невідома функція. Відносно неї дістанемо рівняння:

$$
\begin{gathered}
\frac{d Z_{1}}{d t}=\left(D_{1}(t, \varepsilon)+\sum_{l=1}^{q} B_{1 l}(t, \varepsilon, \theta) \mu^{l}\right) Z_{1}-Z_{1}\left(D_{2}(t, \varepsilon)+\sum_{l=1}^{q} B_{2 l}(t, \varepsilon, \theta) \mu^{l}\right)+ \\
+\varepsilon K_{1}(t, \varepsilon, \theta, \mu)+\mu^{2 q} C_{1}(t, \varepsilon, \theta, \mu)+\varepsilon H_{3}(t, \varepsilon, \theta, \mu) Z_{1}-\varepsilon Z_{1} H_{4}(t, \varepsilon, \theta, \mu)+ \\
\quad+\mu^{q+1}\left(R_{3}(t, \varepsilon, \theta, \mu) Z_{1}-Z_{1} R_{4}(t, \varepsilon, \theta, \mu)\right)+\mu \Phi_{3}\left(t, \varepsilon, \theta, Z_{1}, \mu\right) .
\end{gathered}
$$

Тепер на підставі леми 3 зводимо це рівняння до вигляду (24).

Лему доведено. 


\section{5. Основні результати.}

Теорема 1. Нехай рівняння (24) таке, що існує $q_{0} \in \mathbb{N}\left(1 \leq q_{0} \leq q\right)$ maке, що

$$
\inf _{G\left(\varepsilon_{0}\right)}\left|\operatorname{Re}\left(\left(U_{1 q_{0}}(t, \varepsilon)\right)_{j j}-\left(U_{2 q_{0}}(t, \varepsilon)\right)_{k k}\right)\right| \geq b_{0}>0(j, k=\overline{1, N}),
$$

$i$ для будъ-якого $l=\overline{1, q_{0}-1}\left(\right.$ якщо $\left.q_{0}>1\right)$ виконано:

$$
\operatorname{Re}\left(\left(U_{1 l}(t, \varepsilon)\right)_{j j}-\left(U_{2 l}(t, \varepsilon)\right)_{k k}\right) \equiv 0(j, k=\overline{1, N}) .
$$

Тодi існують $\mu_{3} \in(0,1), \varepsilon_{1}(\mu) \in\left(0, \varepsilon_{0}\right)$ такі, що для будь-яких $\mu \in\left(0, \mu_{3}\right)$, $\varepsilon \in\left(0, \varepsilon_{1}(\mu)\right)$ рівняння (24) має частинний розв'язок, що належить до класу $F_{2}\left(m-1 ; \varepsilon_{1}(\mu) ; \theta\right)$.

Доведення. Будемо виходити з твердження леми 4. Здійснимо в рівнянні (24) підстановку

$$
Z=\frac{\varepsilon+\mu^{2 q}}{\mu^{q_{0}}} \widetilde{Z},
$$

де $\widetilde{Z}$ - нова невідома матриця-функція. Дістанемо:

$$
\begin{gathered}
\frac{d \widetilde{Z}}{d t}=\left(D_{1}(t, \varepsilon)+\sum_{l=1}^{q} U_{1 l}(t, \varepsilon) \mu^{l}\right) \widetilde{Z}-\widetilde{Z}\left(D_{2}(t, \varepsilon)+\sum_{l=1}^{q} U_{2 l}(t, \varepsilon) \mu^{l}\right)+ \\
+\frac{\varepsilon \mu^{q_{0}}}{\varepsilon+\mu^{2 q}} K(t, \varepsilon, \theta, \mu)+\frac{\mu^{2 q+q_{0}}}{\varepsilon+\mu^{2 q}} C(t, \varepsilon, \theta, \mu)+\varepsilon V_{1}(t, \varepsilon, \theta, \mu) \widetilde{Z}-\varepsilon \widetilde{Z} V_{2}(t, \varepsilon, \theta, \mu)+ \\
+\mu^{q+1}\left(R_{1}(t, \varepsilon, \theta, \mu) \widetilde{Z}-\widetilde{Z} R_{2}(t, \varepsilon, \theta, \mu)\right)+\frac{\varepsilon+\mu^{2 q}}{\mu^{q_{0}-1}} \widetilde{\Phi}_{2}(t, \varepsilon, \theta, \widetilde{Z}, \mu) .
\end{gathered}
$$

Розглянемо відповідне лінійне неоднорідне рівняння:

$$
\begin{gathered}
\frac{d \widetilde{Z}_{0}}{d t}=\left(D_{1}(t, \varepsilon)+\sum_{l=1}^{q} U_{1 l}(t, \varepsilon) \mu^{l}\right) \widetilde{Z}_{0}-\widetilde{Z}_{0}\left(D_{2}(t, \varepsilon)+\sum_{l=1}^{q} U_{2 l}(t, \varepsilon) \mu^{l}\right)+ \\
+\frac{\varepsilon \mu^{q_{0}}}{\varepsilon+\mu^{2 q}} K(t, \varepsilon, \theta, \mu)+\frac{\mu^{2 q+q_{0}}}{\varepsilon+\mu^{2 q}} C(t, \varepsilon, \theta, \mu) .
\end{gathered}
$$

На підставі леми 2 з роботи [7] можна зробити висновок, що рівняння (35) має єдиний частинний розв'язок, що належить до класу $F_{2}\left(m-1 ; \varepsilon_{0} ; \theta\right)$, причому існує $T_{1} \in(0,+\infty)$ таке, що

$$
\begin{gathered}
\left\|\widetilde{Z}_{0}(t, \varepsilon, \theta, \mu)\right\|_{F_{2}\left(m-1 ; \varepsilon_{0} ; \theta\right)} \leq \\
\leq \frac{T_{1}}{b_{0} \mu^{q_{0}}}\left(\frac{\varepsilon \mu^{q_{0}}}{\varepsilon+\mu^{2 q}}\|K(t, \varepsilon, \theta, \mu)\|_{F_{2}\left(m-1 ; \varepsilon_{0} ; \theta\right)}+\frac{\mu^{2 q+q_{0}}}{\varepsilon+\mu^{2 q}}\|C(t, \varepsilon, \theta, \mu)\|_{F_{2}\left(m-1 ; \varepsilon_{0} ; \theta\right)}\right)< \\
<\frac{T_{1}}{b_{0}}\left(\|K(t, \varepsilon, \theta, \mu)\|_{F_{2}\left(m-1 ; \varepsilon_{0} ; \theta\right)}+\|C(t, \varepsilon, \theta, \mu)\|_{F_{2}\left(m-1 ; \varepsilon_{0} ; \theta\right)}\right)
\end{gathered}
$$

Розв'язок класу $F_{2}\left(m-1 ; \varepsilon_{1}(\mu) ; \theta\right)$ рівняння (34) шукатимемо методом послідовних наближень, обираючи в якості початкового наближення $\widetilde{Z}_{0}(t, \varepsilon, \theta, \mu)$, а 
подальші наближення визначивши як розв'язки класу $F_{2}\left(m-1 ; \varepsilon_{0} ; \theta\right)$ лінійних неоднорідних матричних рівнянь:

$$
\begin{aligned}
& \frac{d \widetilde{Z}_{\nu+1}}{d t}=\left(D_{1}(t, \varepsilon)+\sum_{l=1}^{q} U_{1 l}(t, \varepsilon) \mu^{l}\right) \widetilde{Z}_{\nu+1}-\widetilde{Z}_{\nu+1}\left(D_{2}(t, \varepsilon)+\sum_{l=1}^{q} U_{2 l}(t, \varepsilon) \mu^{l}\right)+ \\
& +\frac{\varepsilon \mu^{q_{0}}}{\varepsilon+\mu^{2 q}} K(t, \varepsilon, \theta, \mu)+\frac{\mu^{2 q+q_{0}}}{\varepsilon+\mu^{2 q}} C(t, \varepsilon, \theta, \mu)+\varepsilon V_{1}(t, \varepsilon, \theta, \mu) \widetilde{Z}_{\nu}-\varepsilon \widetilde{Z}_{\nu} V_{2}(t, \varepsilon, \theta, \mu)+ \\
& +\mu^{q+1}\left(R_{1}(t, \varepsilon, \theta, \mu) \widetilde{Z}_{\nu}-\widetilde{Z}_{\nu} R_{2}(t, \varepsilon, \theta, \mu)\right)+\frac{\varepsilon+\mu^{2 q}}{\mu^{q_{0}-1}} \widetilde{\Phi}_{2}\left(t, \varepsilon, \theta, \widetilde{Z}_{\nu}, \mu\right) .
\end{aligned}
$$

Нехай

$$
\Omega=\left\{\widetilde{Z} \in F_{2}\left(m-1 ; \varepsilon_{0} ; \theta\right):\left\|\widetilde{Z}-\widetilde{Z}_{0}\right\|_{F_{2}\left(m-1 ; \varepsilon_{0} ; \theta\right)} \leq \rho\right\} .
$$

Позначимо:

$$
\begin{gathered}
M(\rho)=\sup _{\widetilde{Z} \in \Omega}\left\|\widetilde{\Phi}_{2}(t, \varepsilon, \theta, \widetilde{Z}, \mu)\right\|_{F_{2}\left(m-1 ; \varepsilon_{0} ; \theta\right)}, \\
V=\max \left(\left\|V_{1}(t, \varepsilon, \theta, \mu)\right\|_{F_{2}\left(m-1 ; \varepsilon_{0} ; \theta\right)},\left\|V_{2}(t, \varepsilon, \theta, \mu)\right\|_{F_{2}\left(m-1 ; \varepsilon_{0} ; \theta\right)}\right), \\
R=\max \left(\left\|R_{1}(t, \varepsilon, \theta, \mu)\right\|_{F_{2}\left(m-1 ; \varepsilon_{0} ; \theta\right)},\left\|R_{2}(t, \varepsilon, \theta, \mu)\right\|_{F_{2}\left(m-1 ; \varepsilon_{0} ; \theta\right)}\right) .
\end{gathered}
$$

3 того, що $\widetilde{\Phi}_{2}$ диференційовна в сенсі Фреше за $\widetilde{Z}$, а область $\Omega$ замкнена і обмежена, випливає, що існує $L(\rho) \in(0,+\infty)$ таке, що для будь-яких $Z, \bar{Z} \in \Omega$ виконано:

$$
\left\|\widetilde{\Phi}_{2}(t, \varepsilon, \theta, Z, \mu)-\widetilde{\Phi}_{2}(t, \varepsilon, \theta, \bar{Z}, \mu)\right\|_{F_{2}\left(m-1 ; \varepsilon_{0} ; \theta\right)} \leq L(\rho)\|Z-\bar{Z}\|_{F_{2}\left(m-1 ; \varepsilon_{0} ; \theta\right)} .
$$

Покажемо, що при певних співвідношеннях між параметрами $\varepsilon$ і $\mu$ всі наближення, що визначаються формулами (37), залишаються всередині області $\Omega$. Очевидно, що $\widetilde{Z}_{0} \in \Omega$. Припустимо за індукцією, що $\widetilde{Z}_{\nu} \in \Omega$, і розглянемо:

$$
\begin{gathered}
\frac{d\left(\widetilde{Z}_{\nu+1}-\widetilde{Z}_{0}\right)}{d t}=\left(D_{1}(t, \varepsilon)+\sum_{l=1}^{q} U_{1 l}(t, \varepsilon) \mu^{l}\right)\left(\widetilde{Z}_{\nu+1}-\widetilde{Z}_{0}\right)- \\
-\left(\widetilde{Z}_{\nu+1}-\widetilde{Z}_{0}\right)\left(D_{2}(t, \varepsilon)+\sum_{l=1}^{q} U_{2 l}(t, \varepsilon) \mu^{l}\right)+\varepsilon V_{1}(t, \varepsilon, \theta, \mu) \widetilde{Z}_{\nu}-\varepsilon \widetilde{Z}_{\nu} V_{2}(t, \varepsilon, \theta, \mu)+ \\
+\mu^{q+1}\left(R_{1}(t, \varepsilon, \theta, \mu) \widetilde{Z}_{\nu}-\widetilde{Z}_{\nu} R_{2}(t, \varepsilon, \theta, \mu)\right)+\frac{\varepsilon+\mu^{2 q}}{\mu^{q_{0}-1}} \widetilde{\Phi}_{2}\left(t, \varepsilon, \theta, \widetilde{Z}_{\nu}, \mu\right) .
\end{gathered}
$$

Оскільки $\widetilde{Z}_{\nu} \in \Omega$, то

$$
\left\|\widetilde{Z}_{\nu}\right\|_{F_{2}\left(m-1 ; \varepsilon_{0} ; \theta\right)} \leq \rho+\left\|\widetilde{Z}_{0}\right\|_{F_{2}\left(m-1 ; \varepsilon_{0} ; \theta\right)} .
$$

На підставі нерівності (36) і внаслідок умов теореми маємо:

$$
\begin{gathered}
\left\|\widetilde{Z}_{\nu+1}-\widetilde{Z}_{0}\right\|_{F_{2}\left(m-1 ; \varepsilon_{0} ; \theta\right)} \leq \\
\leq \frac{T_{1}}{b_{0} \mu^{q_{0}}}\left[2^{m+1}\left(\varepsilon V+\mu^{q+1} R\right)\left(\rho+\left\|\widetilde{Z}_{0}\right\|_{F_{2}\left(m-1 ; \varepsilon_{0} ; \theta\right)}\right)+\frac{\varepsilon+\mu^{2 q}}{\mu^{q_{0}-1}} M(\rho)\right] .
\end{gathered}
$$

Розділ 1: Математика і статистика 
Отже, при виконанні умови

$$
\frac{T_{1}}{b_{0} \mu^{q_{0}}}\left[2^{m+1}\left(\varepsilon V+\mu^{q+1} R\right)\left(\rho+\left\|\widetilde{Z}_{0}\right\|_{F_{2}\left(m-1 ; \varepsilon_{0} ; \theta\right)}\right)+\frac{\varepsilon+\mu^{2 q}}{\mu^{q_{0}-1}} M(\rho)\right] \leq \rho_{0}<\rho
$$

потрібну належність забезпечено.

Доведемо тепер збіжність процесу, що визначається формулами (37), до розв’язку класу $F_{2}\left(m-1 ; \varepsilon_{1}(\mu) ; \theta\right)$ рівняння (34). Розглянемо:

$$
\begin{gathered}
\frac{\left(\widetilde{Z}_{\nu+1}-\widetilde{Z}_{\nu}\right)}{d t}=\left(D_{1}(t, \varepsilon)+\sum_{l=1}^{q} U_{1 l}(t, \varepsilon) \mu^{l}\right)\left(\widetilde{Z}_{\nu+1}-\widetilde{Z}_{\nu}\right)- \\
-\left(\widetilde{Z}_{\nu+1}-\widetilde{Z}_{\nu}\right)\left(D_{2}(t, \varepsilon)+\sum_{l=1}^{q} U_{2 l}(t, \varepsilon) \mu^{l}\right)+ \\
+\varepsilon V_{1}(t, \varepsilon, \theta, \mu)\left(\widetilde{Z}_{\nu}-\widetilde{Z}_{\nu-1}\right)-\varepsilon\left(\widetilde{Z}_{\nu}-\widetilde{Z}_{\nu-1}\right) V_{2}(t, \varepsilon, \theta, \mu)+ \\
+\mu^{q+1}\left(R_{1}(t, \varepsilon, \theta, \mu)\left(\widetilde{Z}_{\nu}-\widetilde{Z}_{\nu-1}\right)-\left(\widetilde{Z}_{\nu}-\widetilde{Z}_{\nu-1}\right) R_{2}(t, \varepsilon, \theta, \mu)\right)+ \\
+\frac{\varepsilon+\mu^{2 q}}{\mu^{q_{0}-1}}\left(\widetilde{\Phi}_{2}\left(t, \varepsilon, \theta, \widetilde{Z}_{\nu}, \mu\right)-\widetilde{\Phi}_{2}\left(t, \varepsilon, \theta, \widetilde{Z}_{\nu-1}, \mu\right)\right) .
\end{gathered}
$$

Звідси і на підставі нерівностей (36) і (38) матимемо:

$$
\begin{gathered}
\left\|\widetilde{Z}_{\nu+1}-\widetilde{Z}_{\nu}\right\|_{F_{2}\left(m-1 ; \varepsilon_{0} ; \theta\right)} \leq \frac{T_{1}}{b_{0} \mu^{q_{0}}}\left[2^{m}\left(\varepsilon V+\mu^{q+1} R\right)\left\|\widetilde{Z}_{\nu}-\widetilde{Z}_{\nu-1}\right\|_{F_{2}\left(m-1 ; \varepsilon_{0} ; \theta\right)}+\right. \\
\left.+\frac{\varepsilon+\mu^{2 q}}{\mu^{q_{0}-1}} L(\rho)\left\|\widetilde{Z}_{\nu}-\widetilde{Z}_{\nu-1}\right\|_{F_{2}\left(m-1 ; \varepsilon_{0} ; \theta\right)}\right] .
\end{gathered}
$$

Отже, при виконанні нерівності

$$
\frac{T_{1}}{b_{0} \mu^{q_{0}}}\left[2^{m}\left(\varepsilon V+\mu^{q+1} R\right)+\frac{\varepsilon+\mu^{2 q}}{\mu^{q_{0}-1}} L(\rho)\right]<1
$$

потрібну збіжність забезпечено. Нерівності (41), (42) виконуються для достатньо малих $\mu, \varepsilon / \mu^{q_{0}}, \varepsilon / \mu^{2 q_{0}-1}$. Оскільки $0<\mu<1$, то $\varepsilon / \mu^{q_{0}} \leq \varepsilon / \mu^{2 q_{0}-1}$, тому достатньо вимоги мализни $\mu$ і відношення $\varepsilon / \mu^{2 q_{0}-1}$. Таким чином $\varepsilon_{1}(\mu)=T_{2} \mu^{2 q_{0}-1}$, де $T_{2}$ достатньо мале.

З урахуванням (33) отримуємо твердження теореми.

Безпосередньо з леми 4 та теореми 1 випливає наступна теорема.

Теорема 2. Нехай рівняння (2) таке, що

1) виконано умови $1^{0}, 2^{0}$;

2) виконано умови леми 2;

3) для рівняння (7), що отримується з рівняння (2) шляхом підстановки (6), виконано умови леми 4;

4) для рівняння (24), що отримуеться з рівняння (7) шляхом підстановки (23), виконано умови теореми 1.

Тодi існують такі $\mu_{4} \in(0,1), \varepsilon_{4}(\mu) \in\left(0, \varepsilon_{0}\right)$, що для будъ-яких $\mu \in\left(0, \mu_{4}\right)$ $i$ будь-яких $\varepsilon \in\left(0, \varepsilon_{4}(\mu)\right)$ рівняння (2) має частинний розв'язок класу $F_{2}\left(m-1 ; \varepsilon_{4}(\mu) ; \theta\right)$.

Наук. вісник Ужгород. ун-ту. 2021. Том 39, № 2 ISSN 2616-7700 (print), 2708-9568 (online) 


\section{Список використаної літератури}

1. Boichuk A. A., Krivosheya S. A. A Critical Periodic Boundary Value Problem for a Matrix Riccati Equation. Differential equations. 2001. Vol. 37, № 4. P. 464-471.

2. Чуйко С. М.Өлементы теории линейных матричных уравнений: монография. Славянск: Вид-во Б. І. Маторіна, 2017. 163 с.

3. Чуйко С. М.О решении обобщённого матричного уравнения Сильвестра. Чебышевский сборник. 2015. Т. 16. Вып. 1. C. 52-66. DOI: https://doi.org/10.22405/2226-8383-2015-16-1$52-66$

4. Чуйко С. М., Несмелова (Старкова) О. В., Сысоев Д. В. Нелинейная матричная краевая задача в случае параметрического резонанса. Компъютерные исследования и моделирование. 2015. Т. 7. № 4. C. 821-833. DOI: https://doi.org/10.20537/2076-7633-2015-7-4-821-833

5. Verde-Star L. On linear matrix differential equations. Advances in Applied Mathematics. 2007. 39. P. 329-344.

6. Щёголев С. А., Карапетров В. В. Об одном классе решений квазилинейных матричных дифференциальных уравнений. Дослідження в математиці $і$ механіці. 2020. Т. 25. Вип. № 2(36). C. 95-102. DOI: https://doi.org/10.18524/2519-206X.2020.2(36).233806.

7. Щоголев С. А., Карапетров В. В. Блочне розщеплення системи лінійних матричних диференціальних рівнянь. Науковий вісник Ужжгородсъкого університету. Серія «Математика і інформатика». 2021. Вип. 38 № 1. C. 94-104. DOI: https://doi.org/10.24144/2616$7700.2021 .38(1) .94-104$

8. Бари Н. К. Тригонометрические ряды: монография. Москва: Физматгиз. 1961. 935 с.

9. Колмогоров А. Н., Фомин С. В. Элементы теории функций и функционального анализа: монография. Москва: Наука. 1972. 496 с.

10. Малкин И. Г. Некоторые задачи теории нелинейных колебаний: монография. Москва, Гостехиздат. 1956. $491 \mathrm{c.}$

Shchogolev S. A., Karapetrov V. V. Block separation of the system of the linear matrix differential equations.

In the mathematical description of various phenomena and processes arising in mathematical physics, electrical engineering, economics, have to deal with matrix differential equations. Therefore, such equations are relevant as for mathematicians and specialists in other fields natural sciences. This article considers quasilinear matrix differential equations with coefficients depicted in the form of absolutely and uniformly convergent Fourier series with slow variable in a sense coefficients and frequency (class $F$ ). The differences of the diagonal elements of the matrices of the linear part are pure imaginary, that is, we are dealing with a critical case. But between these diagonal elements assume certain relations that indicate the absence of resonance between the natural frequencies of the system and frequency of external excitation force. The problem is considered establishing signs of existence in such an equation of class solutions $F$. By means of a number of transformations the equation is reduced to the equation noncritical case, and the solution of the class $F$ of this equation is sought by the method of successive approximations using the principle compression reflections. Then based on the properties of the solutions of the transformed equation, conclusions are drawn about the properties initial equation.

Keywords: matrix differential equations, Fourier series, slowly varying parameters.

\section{References}

1. Boichuk, A. A, \& Krivosheya, S. A. (2001). A Critical Periodic Boundary Value Problem for a Matrix Riccati Equation. Differential equations, 37(4), 464-471.

2. Chuiko, S. M. (2017). Elementy teorii lineynyih matrichnyh uravneniy [Elements of the theory of linear matrix equations]. Slavyansk [in Russian].

3. Chuiko, S. M. (2015). O reshenii obobshchyonnogo matrichnogo uravneniya Silvestra [On the solutions of the generalized matrix equations of Sylvestr]. Chebyshevsky sbornik, 16(1), 52-66. https://doi.org/10.22405/2226-8383-2015-16-1-52-66 [in Russian].

Розділ 1: Математика і статистика 
4. Chuiko, S. M., Nesmelova (Starkova), O. V., \& Sysoev, D. V. (2015). Nelinejnaya matrichnaya kraevaya zadacha $\mathrm{v}$ sluchae parametricheskogo rezonansa. [Nonlinear boudary value problem in the case of parametric resonance]. Computer Research and Modeling, 7(4), 821-833. https://doi.org/10.20537/2076-7633-2015-7-4-821-833 [in Russian].

5. Verde-Star, L. (2007). On linear matrix differential equations. Advances in Applied Mathematics, 39, 329-344.

6. Shchogolev, S. A., \& Karapetrov, V. V. (2020). Ob odnom klasse reshenij kvazilinejnyh matrichnyh differencial'nyh uravnenij [On one class of solutions of the quasilinear matrix differential equations]. Researches in Mathematics and Mechanics, 25, 2(36), 95-102. https://doi.org/10.18524/2519-206X.2020.2(36).233806. [in Russian].

7. Shchogolev, S. A., \& Karapetrov, V. V. (2021). Block separation of the system of the linear matrix differential equations. Scientific Bulletin of Uzhhorod University. Series of Mathematics and Informatics, 38(1), 94-104. https://doi.org/10.24144/2616-7700.2021.38(1).94-104 [in Ukrainian].

8. Bari, N. K. (1961). Triginometrichskye ryady [Trigonometric series]. Moskva: Fizmatgiz [in Russian].

9. Kolmogorov, A. N, \& Fomin, S. V. (1972). Functsionalnyi analiz [Functional analysis]. Moskva: Nauka [in Russian].

10. Malkin, I. G. (1956). Nekotorye zadachi teorii nelinejnyh kolebanij [Some problems of the theory of nonlinear oscillations]. Moskva: Gostekhizdat [in Russian].

Одержано 23.09.2021 